\title{
Failed Back Surgery Syndrome: A Review Article
}

\author{
James R. Daniell ${ }^{1,2}$, Orso L. Osti ${ }^{1,2}$ \\ ${ }^{1}$ The University of Adelaide, Adelaide, SA, Australia \\ ${ }^{2}$ Calvary Health Care, North Adelaide Campus, North Adelaide, SA, Australia
}

Postsurgical spine syndrome is becoming an increasingly common challenge for clinicians who deal with spinal disorders owing to the expanding indications for spinal surgery and the aging world population. A multidisciplinary approach is most appropriate for patients who are unlikely to benefit from further formal surgical intervention. Anticonvulsant medications are effective in managing neuropathic pain after surgery, whereas opioids are rarely beneficial. Neuromodulation via a surgically implanted dorsal column neurostimulator is gaining popularity owing to its substantial superiority over conventional medical management and/or further surgical intervention. However, considering that prevention is always better than cure, spinal surgeons need to be well aware of the many poor prognostic indicators for spinal surgery, particularly psychosocial overlay.

Keywords: Postsurgical spine syndrome; Failed back surgery syndrome; Repeat spinal surgery; Chronic pain management; Neuromodulation; Spinal cord stimulation

\section{Introduction}

Low back pain (LBP) has been estimated to have a lifetime prevalence of $60 \%-80 \%$ among the global population, making it one of the most common health complaints [1]. Approximately $10 \%$ of individuals suffering from LBP have symptoms that persist for longer than 3 months [2]. As a consequence of the large number of patients with LBP who have sought treatment, a substantial increase in those undergoing surgery has been observed over the past two decades.

Failed back surgery syndrome (FBSS) is a term used to describe a clinical entity that has been acknowledged since the advent of spinal surgery. It was perhaps best described by Follett and Dirks [3] as the "surgical end stage after one or several interventions on the lumbar neuroaxis indicated to relieve lower back pain, radicular pain or the combination of both, without effect". A more functional definition is "when the outcome of lumbar spinal surgery does not meet the pre-surgical expectations of the patient and surgeon" [4].

The term FBSS has been criticized for being a clinical misnomer for both patients and surgeons alike [5]. The qualifier "failed" does little to elucidate the entity, and it is perhaps most appropriate to abandon this term entirely. The diagnostic term "postsurgical spine syndrome" perhaps more accurately describes the aforementioned clinical entity [6].

Between 1998 and 2008, the yearly number of lumbar fusion surgeries performed in the United States increased from 77,682 to 210,407 , with the total number of spinal operations exceeding one million in 2002 [7,8]. The direct yearly cost of spinal fusion surgery in the United States was over US\$ 16 billion in 2004 [9], whereas the overall

Received Mar 2, 2017; Revised Mar 3, 2017; Accepted May 28, 2017

Corresponding author: Orso L. Osti

Calvary Health Care, 89 Strangways Terrace, North Adelaide, SA 5006, Australia

E-mail: orsolosti@gmail.com 
failure rate of lumbar spine surgery was estimated to be $10 \%-46 \%$ [10]. Given that these rates have not changed substantially over the years despite advances in technology and surgical technique, the number of patients developing FBSS can be expected to continually increase [11].

The potential widespread occurrence of this condition necessitates accurate assessment of this challenging patient population to best address their symptoms and deliver the most effective treatment.

\section{The Problem}

Repeat spinal surgery is a treatment option with diminishing returns. Although more than $50 \%$ of primary spinal surgeries are successful, no more than $30 \%, 15 \%$, and $5 \%$ of the patients experience a successful outcome after the second, third, and fourth surgeries, respectively [12]. The prevalence and incidence of patients with FBSS are comparable with those of patients with rheumatoid arthritis. However, patients with FBSS and neuropathic pain experience higher levels of pain and a poorer quality of life and physical function compared with those with osteoarthritis, rheumatoid arthritis, complex regional pain syndrome, and fibromyalgia [13].

\section{Factors Leading to Failed Back Surgery Syndrome}

\section{Patient factors}

A number of patient-related factors may be associated with higher rates of FBSS and should be considered when discussing treatment options. A patient's psychosocial wellbeing has a significant effect on his/her treatment outcome and pain experience such that Carragee et al. [14] found poor psychosocial wellbeing to be the strongest predictor of LBP disability in a study comparing morphological and social risk factors. This is also true when predicting poor outcomes after spinal surgery [15]. A large number of patients assessed for spinal surgery comprise those claiming workers' compensation or receiving disability support pension. Both of these groups have been found to be significantly more likely to have poorer outcomes after spinal surgery, with those claiming workers' compensation having the poorest outcomes. The figures become even more alarming after considering preexisting depression [16].
Furthermore, smokers are more likely to experience poorer outcomes after spinal surgery [17], and obesity is an established predictor of higher rates of postoperative complications [18].

\section{Operative factors}

A percentage of patients who had undergone spinal surgery may have experienced poor outcomes due to an inappropriate surgical choice. As previously mentioned, the number of prior spinal surgeries is a significant predictor of outcome of the following spinal surgery.

A single-level decompressive laminectomy in the presence of unknown multi-level spinal involvement is unlikely to relieve the patient's presenting symptoms. Similarly, decompressive surgery in the presence of predominantly axial/mechanical pain is unlikely to lead to a satisfactory outcome. Furthermore, the most commonly reported error during spinal surgery was wrong-level decompression [19].

Intraoperative errors during spinal surgery may not only cause ongoing pain in the same preoperative distribution site but also trigger new pain sources. Each surgery has the potential to create new segmental instability and generate further pain with misplaced interbody fusion grafts and implants, which may, for example, potentially cause neural impingement [19]. In an analysis of 105 lumbar fusion cases with pedicle screw instrumentation, the incidence rates of screw placement errors and implant breakage at follow-up were $6.5 \%$ and $12.0 \%$, respectively, with potential for serious neurological complications [20].

Patients undergoing lumbar fusion are at risk of loss of sagittal balance, particularly when more than one level is involved. Furthermore, those with reduced sacral tilt after surgery have been found to exhibit loss of natural lumbar lordosis with a resultant increase in stress transfer to the sacroiliac joints [21].

\section{Postoperative factors}

1) Early

Immediately following surgery, a number of factors may lead to operative failure. Procedural complications that may have deleterious effects on the outcome include hematoma formation in the epidural or subdural space, infection, pseudomeningocele, and nerve injury. 


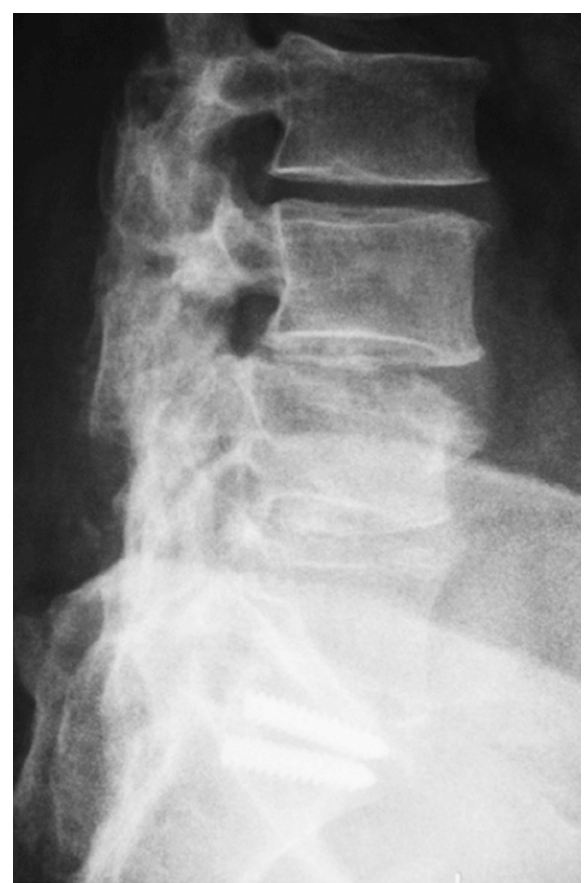

Fig. 1. A typical radiological example of "failed back surgery syndrome": previous $L 4$ to S1 posterolateral fusion with adjacent (L3-L4) segment degeneration, residual/recurrent neural involvement, broken screw instrumentation, and loss of lumbar lordosis.

\section{2) Late}

Altered biomechanics in the operated spine can potentially shift segmental stress and cause "transition syndrome". Changes in load distribution may accelerate degeneration in segments adjacent to the lumbar fusion, which could lead to new sources of pain. One long-term radiological study reported that $36 \%$ of the cases developed "transition syndrome" 5 years after lumbar fusion [22,23] (Fig. 1). Postlumbar discectomy patients with one-level disc herniation have been reported to have an overall recurrence rate of $5 \%-25 \%$ within 5 years.

\section{Assessment}

In the workup of patients with FBSS, the principal clinician involved should be able to utilize the services of a multidisciplinary team.

\section{History}

First and foremost, the patient's psychosocial wellbeing should be thoroughly assessed. The presence of any psychiatric comorbidity, workers' compensation claims, or personal disability claims should be noted. These factors should be addressed during treatment and should not be used to exclude patients from further treatment.

An accurate and detailed pain history is crucial in delineating the likely source of the patient's pain. The difference between predominantly axial pain and neuropathic pain or the presence of both should be understood. Similarities in the distribution of prior and current pain are also highly relevant given that new sources of pain may be an indication of unrelated pathologies [24].

It is always of great importance to eliminate any "red flags" in the history that may necessitate urgent intervention and investigation, including signs of any bowel or bladder disturbance, any new or progressive neurological deficit, signs of possible infection, and weight loss or other symptoms that may indicate an undiagnosed malignancy. Thorough documentation of previous treatments should be included during history taking.

\section{Physical examination}

Physical examination of patients with FBSS is mainly performed to eliminate the presence of any ominous diagnoses that may have been suggested in the past. In fact, published studies show that very few clinical signs have a high predictive value in identifying the source of pain [24]. Although paraspinal tenderness has been shown to be associated with facet arthropathy, the specificity of this test is low [25]. A neurological examination should be performed to confirm the absence of progressive neurological deficit and to establish a baseline prior to any further intervention.

\section{Investigations}

The choice of an imaging modality for the FBSS cohort is dependent on the underlying presumptive diagnosis and previous treatments.

Plain erect radiographs are of use in the initial assessment of vertebral alignment and the extent of previous surgical intervention as well as in detecting substantial and clinically significant degenerative changes [19]. Plain lateral dynamic erect radiographs (with extension/flexion views) have been shown to be superior to magnetic resonance imaging (MRI) in detecting spondylolisthesis [26].

However, MRI remains the gold standard in FBSS owing to its superior contrast enhancement sensitivity 
compared with computed tomography (CT) [27]. MRI with gadolinium has the capacity to distinguish between epidural fibrosis, perineural scar tissue, and recurrent disc herniation as a source of pain $[19,25]$ while being the best imaging modality for detecting spondylodiscitis [27].

CT is used when MRI is contraindicated owing to the presence of metalware or non-MRI-compatible cardiac pacemakers, wherein old-fashioned water-soluble CTmyelography may be used [24]. CT may also be used in identifying facet arthropathy and pseudoarthrosis owing to its fine slice superiority in osseous imaging [28].

\section{Management}

\section{Conservative}

Conservative management should always be attempted in patients with FBSS who do not require urgent surgery. Moreover, it should always involve supervised exercise programs with the dual purpose of improving patients' core strength and spinal range of motion, which may include physical therapy and pharmacological management.

Pharmacological therapy may include paracetamol and nonsteroidal anti-inflammatory drugs for axial pain considering that they have both been shown to be superior to placebo in reducing LBP [29]. Anticonvulsant drugs have gained popularity for neuropathic pain, with gabapentin (Neurontin) and pregabalin (Lyrica) being the most commonly used preparations. Gabapentin has been shown to be superior to naproxen in alleviating back and leg pain after spinal surgery [30]. Pregabalin plays a role in the prevention of pain before and after surgery, with its effect apparently increasing with time [31].

The use of opioids in chronic LBP has become increasingly controversial and is currently recommended for only short-term therapy. In fact, opioid treatment should be limited to a finite course over a few weeks given the absence of evidence to suggest any long-term pain improvement from its use [32]. Furthermore, mounting evidence has shown substantial morbidity risks associated with long-term opioid use, including addiction, dependence, overdose, and even death [25,32].

As part of the treatment regimen, some evidence suggests that cognitive behavioral therapy leads to a reduction in pain scores in the immediate postoperative period and during long-term disability [33]. Physical therapy may be used as part of a multimodal approach for pain management given its mild effectiveness in patients with chronic LBP. However, no consensus exists on the best type of therapy [34].

\section{Repeat surgery}

Choosing the most appropriate management modality should be based on the type and pattern of pain syndrome experienced by the patient: those suffering from predominantly axial or mechanical pain and those with predominantly neuropathic lower limb pain.

As mentioned earlier, very few absolute indications exist for repeat spinal surgery. These include any disabling and progressive neurological deficit, be it association with bowel or bladder function impairment, cauda equina syndrome, or established spinal instability requiring reoperation [35].

Removal of pedicle screw instrumentation may be considered during predominantly axial or midline pain after lumbar fusion. In fact, a number of such cases showed a significant reduction in pain scores and opioid requirement after implant removal [36]. It is also important to consider the presence of significant adjacent segment disease or periprosthetic loosening, which may contribute to the pain, as well as underlying osteoporosis requiring appropriate medical management (Fig. 2).

\section{Neuromodulation}

Spinal cord stimulation (SCS) has been proven to be the most effective form of semi-invasive treatment in patients with predominantly neuropathic limb pain. The PROCESS study, published by Kumar et al. [37] in 2007, compared conventional medical management (CMM) alone with SCS+CMM in patients with radicular pain for 6 months following a minimum of one anatomically successful procedure for herniated lumbar nucleus pulposus. After 6 months, the percentage of patients experiencing at least a $50 \%$ reduction in pain scores was $9 \%$ in the CMM alone group against $48 \%$ in the CMM+SCS group [37]. North et al. [38] in 2005 conducted a randomized study comparing SCS with reoperation in patients experiencing radicular pain with or without LBP. In the study, success was defined as a greater than $50 \%$ reduction in pain and satisfaction with treatment; these endpoints were the same as those used in previous similar studies. SCS was more successful than reoperation (nine of 19 patients ver- 

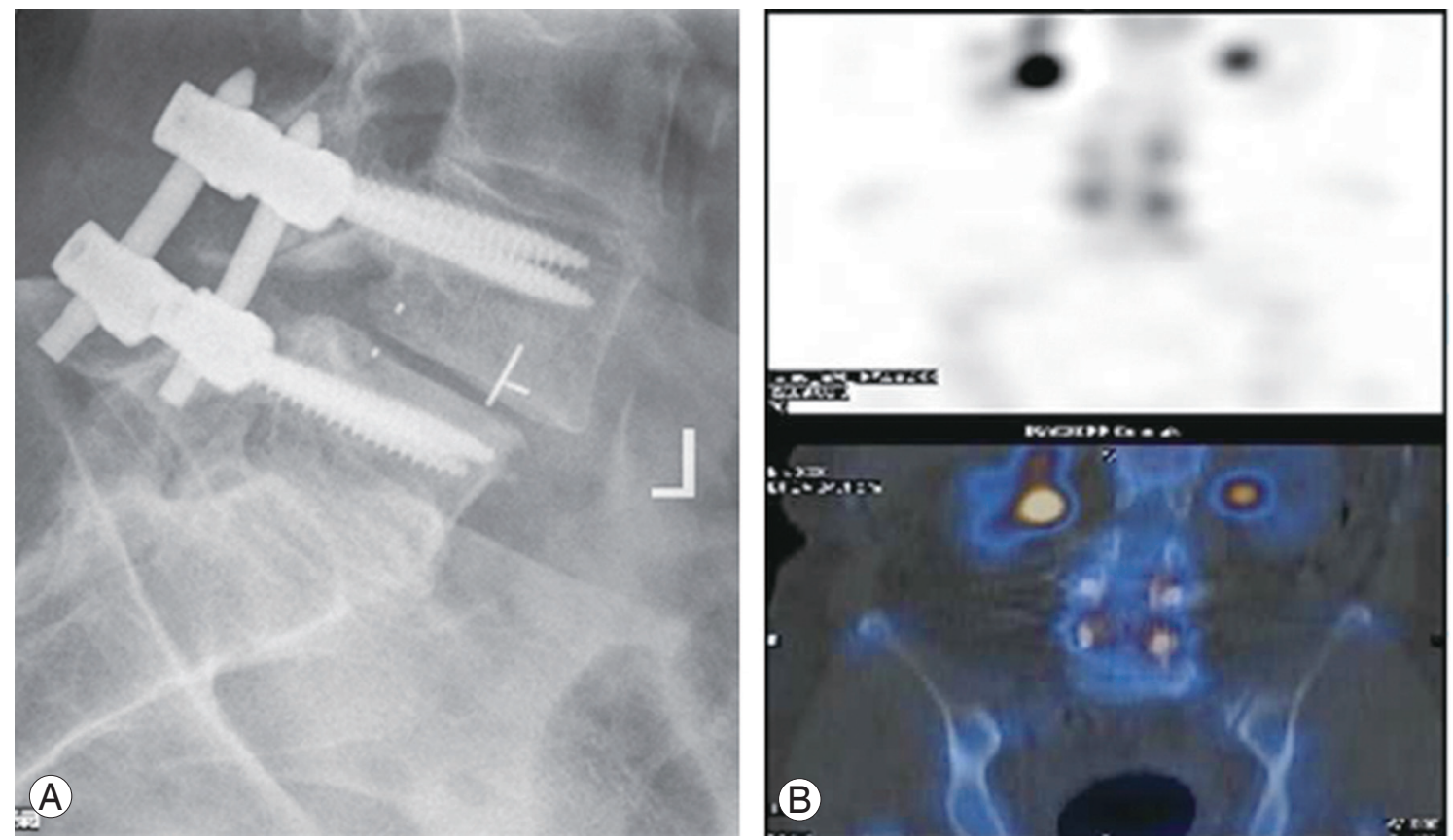

Fig. 2. (A, B) Lucency and loosening surrounding both $L 4$ pedicle screw implants 18 months postsurgery with the diagnostic appearance on bone scan likely to be linked to failure of bony ingrowth into the carbon fiber interbody cage used to replace the $L 4-\mathrm{L} 5$ disc.

sus three of $26, p<0.01)$, whereas fewer patients changed groups from SCS to reoperation [38]. A retrospective analysis of 16,455 patients with FBSS who underwent either reoperation or SCS implantation demonstrated that those with SCS implantation experienced less than half the complications compared with those who underwent reoperation after 90 days (6.5\% versus 14.4\%) [39].

The role of SCS in patients with predominantly axial pain has previously been considered much less promising than that in patients with radicular pain. A recent multicenter randomized controlled trial conducted by Kapural et al. [40] randomized patients with chronic LBP and/ or limb pain to high-frequency SCS $(10,000 \mathrm{~Hz})(\mathrm{HF} 10)$ or traditional low-frequency SCS $(50 \mathrm{~Hz})$. Majority of these patients ( $87 \%$ and $86.2 \%$, respectively) had undergone previous spinal surgery. The primary endpoint of the study was a minimum pain reduction of $50 \%$ without stimulation-related neurological deficits [40]. The trial showed that HF10 was superior to traditional SCS in the treatment of both axial and radicular pain, with HF10 having a better response rate (84.3\%) compared with traditional SCS (43.8\%) in patients with axial pain after 3 months. Among those with radicular pain, HF10 and traditional SCS had response rates of $83.1 \%$ and $55.0 \%$ after 3 months of follow-up, respectively [40]. The superior

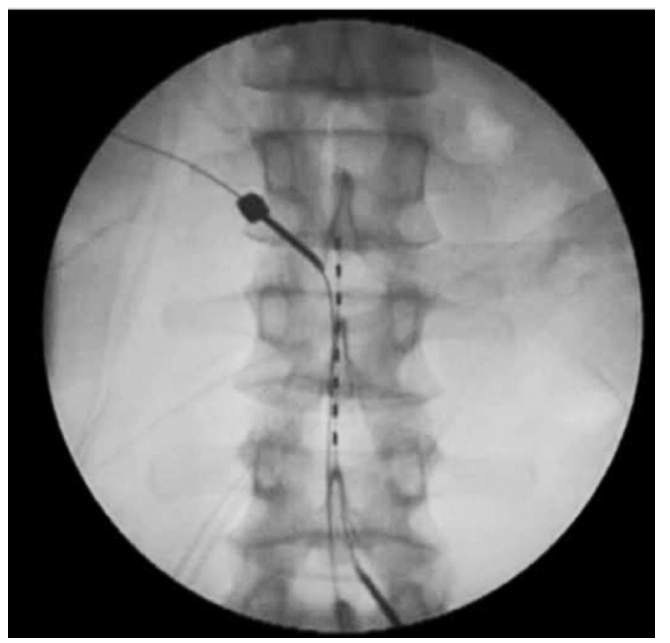

Fig. 3. Example of percutaneous placement of electrodes for the management of persisting axial and neuropathic pain with direct stimulation of the $L 4$ dorsal root ganglion.

outcomes of HF10 therapy continued until 24 months in those with both back and leg pain [41]. The efficacy of the HF10 therapy has also been confirmed in an Australian cohort, with high trial success rates and significant pain reduction among patients who failed to respond to traditional SCS [42].

A number of other neuromodulation techniques have also been attempted, including burst, adaptive, dorsal root 
ganglion, and peripheral nerve field stimulation. Based on one study conducted by Schu et al. [43], only moderate evidence exists for burst stimulation, which demonstrated lower pain scores and higher patient satisfaction over 1 week compared with traditional tonic-clonic stimulation [44]. A prospective study by Liem et al. [45] showed that dorsal root ganglion stimulation had similar efficacy for both leg and back pain in patients with FBSS, although the most significant reduction was observed for foot pain (Fig. 3).

Screening patients for SCS implantation requires comprehensive consultation wherein formal surgery is confirmed to have no further benefit and the patient receives optimal medical management, has realistic insight into their condition, and has no evidence of infection. In general, most trials involving SCS include a trial period of minimum 10 days with at least a $50 \%$ reduction in pain scores. The surgical implantation of leads is associated with greater efficacy during the trial period. It is also worth considering that significantly higher rates of infection have been observed when using externalized leads during the trial.

\section{Conclusions}

Our approach to manage FBSS considers the limitations of surgical intervention on the spine, as well as the various patient-related factors that may lead to unsuccessful outcomes besides the presence of surgically ameliorable pathology. The decision to perform surgery in patients with predominantly axial pain should be made with the understanding that many patients may not respond to the treatment.

The importance of a competent multidisciplinary team in FBSS cannot be overstated. Engagement between physicians, psychologists, physiotherapists, and other allied health professionals is essential in improving outcomes for patients with FBSS.

SCS continues to establish itself as the preferred treatment option for patients with FBSS in the absence of a viable surgical alternative, particularly when radicular/ neuropathic pain, as well as often when substantial axial pain, is the main source of disability.

\section{Conflict of Interest}

No potential conflict of interest relevant to this article was reported.

\section{References}

1. Hoy D, March L, Brooks P, et al. The global burden of low back pain: estimates from the Global Burden of Disease 2010 study. Ann Rheum Dis 2014;73:968-74.

2. Airaksinen O, Brox JI, Cedraschi C, et al. Chapter 4. European guidelines for the management of chronic nonspecific low back pain. Eur Spine J 2006;15 Suppl 2:S192-300.

3. Follett KA, Dirks BA. Etiology and evaluation of the failed back surgery syndrome. Neurosurg Q 1993;3:40-59.

4. Waguespack A, Schofferman J, Slosar P, Reynolds J. Etiology of long-term failures of lumbar spine surgery. Pain Med 2002;3:18-22.

5. Lucas AJ. Failed back surgery syndrome: whose failure?: time to discard a redundant term. Br J Pain 2012;6:162-5.

6. Ordia J, Vaisman J. Post-surgical spine syndrome. Surg Neurol Int 2011;2:132.

7. Rajaee SS, Bae HW, Kanim LE, Delamarter RB. Spinal fusion in the United States: analysis of trends from 1998 to 2008. Spine (Phila Pa 1976) 2012;37:6776.

8. Deyo RA, Gray DT, Kreuter W, Mirza S, Martin BI. United States trends in lumbar fusion surgery for degenerative conditions. Spine (Phila Pa 1976) 2005;30:1441-5.

9. Deyo RA. Back surgery: who needs it? N Engl J Med 2007;356:2239-43.

10. Thomson S. Failed back surgery syndrome: definition, epidemiology and demographics. Br J Pain 2013;7:56-9.

11. Burton CV. Failed back surgery patients: the alarm bells are ringing. Surg Neurol 2006;65:5-6.

12. Nachemson AL. Evaluation of results in lumbar spine surgery. Acta Orthop Scand Suppl 1993;251:130-3.

13. Thomson S, Jacques L. Demographic characteristics of patients with severe neuropathic pain secondary to failed back surgery syndrome. Pain Pract 2009;9:20615.

14. Carragee EJ, Alamin TF, Miller JL, Carragee JM. Discographic, MRI and psychosocial determinants of low back pain disability and remission: a prospective study in subjects with benign persistent back pain. 
Spine J 2005;5:24-35.

15. Voorhies RM, Jiang X, Thomas N. Predicting outcome in the surgical treatment of lumbar radiculopathy using the Pain Drawing Score, McGill Short Form Pain Questionnaire, and risk factors including psychosocial issues and axial joint pain. Spine J 2007;7:516-24.

16. Gum JL, Glassman SD, Carreon LY. Is type of compensation a predictor of outcome after lumbar fusion? Spine (Phila Pa 1976) 2013;38:443-8.

17. Sanden B, Forsth P, Michaelsson K. Smokers show less improvement than nonsmokers two years after surgery for lumbar spinal stenosis: a study of 4555 patients from the Swedish spine register. Spine (Phila Pa 1976) 2011;36:1059-64.

18. Marquez-Lara A, Nandyala SV, Sankaranarayanan S, Noureldin M, Singh K. Body mass index as a predictor of complications and mortality after lumbar spine surgery. Spine (Phila Pa 1976) 2014;39:798-804.

19. Guyer RD, Patterson M, Ohnmeiss DD. Failed back surgery syndrome: diagnostic evaluation. J Am Acad Orthop Surg 2006;14:534-43.

20. Jutte PC, Castelein RM. Complications of pedicle screws in lumbar and lumbosacral fusions in 105 consecutive primary operations. Eur Spine J 2002;11:594-8.

21. Lazennec JY, Ramare S, Arafati N, et al. Sagittal alignment in lumbosacral fusion: relations between radiological parameters and pain. Eur Spine J 2000;9:4755.

22. Kumar MN, Baklanov A, Chopin D. Correlation between sagittal plane changes and adjacent segment degeneration following lumbar spine fusion. Eur Spine J 2001;10:314-9.

23. Hilibrand AS, Carlson GD, Palumbo MA, Jones PK, Bohlman HH. Radiculopathy and myelopathy at segments adjacent to the site of a previous anterior cervical arthrodesis. J Bone Joint Surg Am 1999;81:51928.

24. Chan CW, Peng P. Failed back surgery syndrome. Pain Med 2011;12:577-606.

25. Baber Z, Erdek MA. Failed back surgery syndrome: current perspectives. J Pain Res 2016;9:979-87.

26. Kizilkilic O, Yalcin O, Sen O, Aydin MV, Yildirim T, Hurcan C. The role of standing flexion-extension radiographs for spondylolisthesis following single level disk surgery. Neurol Res 2007;29:540-3.
27. Van Goethem JW, Parizel PM, Jinkins JR. Review article: MRI of the postoperative lumbar spine. Neuroradiology 2002;44:723-39.

28. Eun SS, Lee HY, Lee SH, Kim KH, Liu WC. MRI versus CT for the diagnosis of lumbar spinal stenosis. J Neuroradiol 2012;39:104-9.

29. Chou R, Huffman LH; American Pain Society; American College of Physicians. Medications for acute and chronic low back pain: a review of the evidence for an American Pain Society/American College of Physicians clinical practice guideline. Ann Intern Med 2007;147:505-14.

30. Khosravi MB, Azemati S, Sahmeddini MA. Gabapentin versus naproxen in the management of failed back surgery syndrome; a randomized controlled trial. Acta Anaesthesiol Belg 2014;65:31-7.

31. Canos A, Cort L, Fernandez Y, et al. Preventive analgesia with pregabalin in neuropathic pain from "failed back surgery syndrome": assessment of sleep quality and disability. Pain Med 2016;17:344-52.

32. Franklin GM; American Academy of Neurology. Opioids for chronic noncancer pain: a position paper of the American Academy of Neurology. Neurology 2014;83:1277-84.

33. Williams AC, Eccleston C, Morley S. Psychological therapies for the management of chronic pain (excluding headache) in adults. Cochrane Database Syst Rev 2012;11:CD007407.

34. Machado LA, de Souza Mv, Ferreira PH, Ferreira ML. The McKenzie method for low back pain: a systematic review of the literature with a meta-analysis approach. Spine (Phila Pa 1976) 2006;31:E254-62.

35. Hussain A, Erdek M. Interventional pain management for failed back surgery syndrome. Pain Pract 2014;14:64-78.

36. Zotti MG, Brumby-Rendell OP, McDonald B, et al. The outcome of pedicle screw instrumentation removal for ongoing low back pain following posterolateral lumbar fusion. J Spine Surg 2015;1:50-6.

37. Kumar K, Taylor RS, Jacques L, et al. Spinal cord stimulation versus conventional medical management for neuropathic pain: a multicentre randomised controlled trial in patients with failed back surgery syndrome. Pain 2007;132:179-88.

38. North RB, Kidd DH, Farrokhi F, Piantadosi SA. Spinal cord stimulation versus repeated lumbosacral spine surgery for chronic pain: a randomized, con- 
trolled trial. Neurosurgery 2005;56:98-106.

39. Lad SP, Babu R, Bagley JH, et al. Utilization of spinal cord stimulation in patients with failed back surgery syndrome. Spine (Phila Pa 1976) 2014;39:E719-27.

40. Kapural L, Yu C, Doust MW, et al. Novel $10-\mathrm{kHz}$ High-frequency Therapy (HF10 Therapy) is superior to traditional low-frequency spinal cord stimulation for the treatment of chronic back and leg pain: the SENZA-RCT Randomized Controlled Trial. Anesthesiology 2015;123:851-60.

41. Kapural L, Yu C, Doust MW, et al. Comparison of 10$\mathrm{kHz}$ high-frequency and traditional low-frequency spinal cord stimulation for the treatment of chronic back and leg pain: 24-month results from a multicenter, randomized, controlled pivotal trial. Neurosurgery 2016;79:667-77.

42. Russo M, Verrills P, Mitchell B, Salmon J, Barnard A, Santarelli D. High frequency spinal cord stimulation at $10 \mathrm{kHz}$ for the treatment of chronic pain: 6-month Australian clinical experience. Pain Physician 2016;19:267-80.

43. Schu S, Slotty PJ, Bara G, von Knop M, Edgar D, Vesper J. A prospective, randomised, double-blind, placebo-controlled study to examine the effectiveness of burst spinal cord stimulation patterns for the treatment of failed back surgery syndrome. Neuromodulation 2014;17:443-50.

44. Grider JS, Manchikanti L, Carayannopoulos A, et al. Effectiveness of spinal cord stimulation in chronic spinal pain: a systematic review. Pain Physician 2016;19:E33-54.

45. Liem L, Russo M, Huygen FJ, et al. One-year outcomes of spinal cord stimulation of the dorsal root ganglion in the treatment of chronic neuropathic pain. Neuromodulation 2015;18:41-8. 